\title{
Prevalence of Persistent Proteinuria in Stable HIV/AIDS Patients and Its Association with HIV Nephropathy
}

\author{
Maria Alina Gomes de Mattos Cavalcante ${ }^{1}$, Sandra Neiva Coelho ${ }^{1}$ and Heloísa Ramos Lacerda ${ }^{2}$ \\ ${ }^{1}$ Division of Nephrology, Federal University of Pernambuco; ${ }^{2}$ Division of Infectious Diseases, Federal University of Pernambuco; Recife, PE, Brazil
}

\begin{abstract}
Human immunodeficiency virus (HIV)-related glomerular disease is a cause of end-stage renal disease, though there is no recent data from Brazil concerning this syndrome. Persistent proteinuria (PPt) is the main marker for glomerular disease, especially levels above $1.5 \mathrm{~g}$. We examined the prevalence of and associated risk factors for PPt, along with the prevalence of HIV-associated nephropathy (HIVAN) among AIDS patients. We interviewed 411 patients who were attended at the HIV/AIDS section of the Clinical Hospital of the Federal University of Pernambuco (Brazil) from January through June 2004. PPt was defined as a positive urine dipstick exam on at least two occasions. The analyzed risk factors were: black race, a low CD4 lymphocyte count ( $<200$ cells $\left./ \mathbf{m m}^{3}\right)$, an HIV RNA level of $>100,000$ copies/mL and patients on highly-active antiretroviral therapy (HAART). The patients were classified according to urinary protein/creatinine ratio $(\mathrm{Up} / \mathrm{Uc})<1.0,1.0-3.0$ and $>3.0$. Patients with $\mathrm{Up} / \mathrm{Uc}>3.0$ were submitted to renal biopsy. Among the 411 HIV/AIDS patients, the mean age was 37 years, $70 \%$ were male, $37.5 \%$ were black, the mean CD4 count was 363 cells $/ \mathrm{mm}^{3}$ ( \pm 95), the mean RNA HIV count was 44,475 copies/mL $( \pm 40,369)$, and $92 \%$ were on HAART. The prevalence of PPt was 5.6\% (95\% CI = 3.6 to $8.3 \%)$, and it was significantly associated with a low CD4 lymphocyte count $(\mathbf{p}<\mathbf{0 . 0 4 8})$. HIVAN was found in just one patient, and two patients improved after HAART.
\end{abstract}

Key-Words: Proteinuria, HIV nephropathy, AIDS.

In 2006, there were 39.5 million people with HIV/AIDS worldwide. In Latin America there were 1.7 million people with HIV/AIDS, while 140,000 new cases were registered in 2006. The estimated prevalence within this region is on the order of $0.5 \%$ [1]. Glomerular involvement in HIV patients has been detected since the beginning of the 80s. Two appraisals published in 1984 represent a landmark in describing glomerular disease within this group [2,3]. Other studies have shown various types of glomerular disturbances in HIV/AIDS patients, including: minimal change disease, mesangial proliferative, membranoproliferative, and membranous glomerulonephritis, IgA nephropathy, lupus-like glomerulonephritis, thrombotic microangiopathy, fibrillary glomerulonephritis and immunotactoid glomerulopathy [4-10]. The classical histological pattern associated with HIV is collapsing focal segmental glomerulosclerosis (collapsing FSGS). The clinical and laboratory characteristics of HIVassociated nephropathy (HIVAN) are severe proteinuria, an absence of arterial hypertension, hematuria and edema, with rapid progression to chronic-renal insufficiency. The kidneys become enlarged and highly echogenic, because of intense interstitial edema/fibrosis and microcystic dilatation of the renal tubules [11,12].

Persistent proteinuria is the principal marker of glomerular disease in HIV/AIDS patients and should be used for early identification of HIVAN [13]. Certain factors

Received on 28 February 2007; revised 16 August 2007.

Address for correspondence: Dr. Maria Alina Gomes de Mattos Cavalcante. Praça Fleming 50, ap1802 - Jaqueira (52050-180) Recife - PE, Brazil. Fone number: (81) 91421442/ 2126 3734. Fax number: (81) 2126 3734. E-mail: alina@hotlink.com.br.

The Brazilian Journal of Infectious Diseases 2007;11(5):456-461. (C) 2007 by The Brazilian Journal of Infectious Diseases and Contexto Publishing. All rights reserved. associated with HIVAN, include: a CD4 lymphocyte count $<200$ cells $/ \mathrm{mm}^{3}$, a viral load of $>100,000$ copies $/ \mathrm{mL}$, black race and co-infection with hepatitis $\mathrm{C}$; these factors also are related to poor prognosis in terms of renal function and to increased mortality [14-17].

The prevalence of HIVAN in Brazil has received scant attention. Lopes at al. evaluated 543 HIV patients in Rio de Janeiro and found six cases of nephritic-range proteinuria (1.1\%). The proteinuric patients were black; four presented a histological pattern compatible with HIVAN [18].

We undertook a prospective evaluation and examined the prevalence of proteinuria and HIVAN in stable patients; most of them were receiving highly-active-antiretroviral therapy (HAART). We also examined the association between these disturbances and black race, CD4 level and HIV-1 viral load.

\section{Materials and Methods}

All patients consecutively attended in the HIV/AIDS Division, University Hospital Federal University of Pernambuco (Brazil) from January through June of 2004 were invited to participate in the study. The study included patients not using antiretrovirals (recently diagnosed or assymtomatic), who comprised a minor group (8\%), and patients already using antiretrovirals (92\%). By means of a questionnaire and medical examination, we collected information concerning sex, age, race (white, dark-skinned and black race), duration of HIV-positive status (in years), how the disease was contracted, if there was hospitalization due to infection during the preceding six months, previous diagnosis of diabetes mellitus, primary hypertension or glomerulonephritis, whether they were being treated with HAART, CD4 lymphocyte counts (flow cytometry method) and viral-load levels (PCR-Amplicor, Roche), minimum detection value: $<400$ copies $/ \mathrm{mL}$ ). We also measured serum- 
creatinine levels (bichromatic method - Dade Behring Laboratories, reference interval: 0.6 to $1.3 \mathrm{mg} / \mathrm{dL}$ ), and a urine dipstick was used (Multistix ${ }^{\circledR}$ 10SG Reagent Strips - Bayer Diagnostic Division, Argentine Industry) to determine proteinuria. Patients with positive-proteinuria tests had a second dipstick test and collected urine on at least two occasions to measure the protein-to-creatinine ratio (Up/Uc). The Up/Uc was conducted by means of the pyrogallol molybdate method (Y. Fujita, I. Mori and S. Kitano, Dade Behring Laboratories). During the second phase of our study, patients with persistent proteinuria were classified according to $\mathrm{Up} / \mathrm{Uc}$ as $<1.0$, between 1.0 and 3.0 and $>3.0$. Those with Up/Uc above 3.0 also had a kidney biopsy. The university’s ethics committee authorized the study, and all participating patients gave their written consent.

The association between the categorized variables was evaluated with the Pearson's chi square test or the Fisher exact test. A level of significance of $5 \%(p \leq 0.05)$ was adopted in the tests.

\section{Results}

The 411 patients had an average age of 37 years, and 289 (70\%) were male. Approximately 154 (37.5\%) were of the black race, 182 (44\%) were dark-skinned and 75 (18\%) white. The average duration of HIV-positive status was 4.7 years. Most of the patients acquired HIV infection by sexual route (68.5\%), with heterosexual exposure in 141 (34\%), male-to-male sex

Table 1. Demographic and clinical characteristics of the HIV/ AIDS patients

\begin{tabular}{|c|c|c|c|}
\hline & $\mathbf{N}$ & $\%$ & Mean \pm DP \\
\hline Number & 411 & 100 & \\
\hline Age (years) & & & $37 \pm 9$ \\
\hline \multicolumn{4}{|l|}{ Sex } \\
\hline Male & 289 & 70 & \\
\hline Female & 122 & 30 & \\
\hline \multicolumn{4}{|l|}{ Race } \\
\hline White & 75 & 18 & \\
\hline Dark skinned & 182 & 44 & \\
\hline Black & 154 & 37.5 & \\
\hline Age at HIV diagnosis (years) & & & 4.7 \\
\hline \multicolumn{4}{|l|}{ HIV contamination } \\
\hline Homosexual exposure & 95 & 23 & \\
\hline Heterosexual exposure & 141 & 34 & \\
\hline Bisexual exposure & 46 & 11 & \\
\hline IV drugs & 4 & 1.0 & \\
\hline Unknown & 124 & 30 & \\
\hline Other & 1 & 0.24 & \\
\hline CD4 cell count (cells/mm3) & & & $363 \pm 95$ \\
\hline HIV RNA count (copies/mL) & & & $4,475 \pm 40,369$ \\
\hline HAART & 379 & 92 & \\
\hline Serum creatinine >1.3mg/dL & 17 & 4.0 & \\
\hline Persistent proteinuria & 23 & 5.6 & \\
\hline
\end{tabular}

exposure in 95 (23\%) and bisexual in 46 (11\%). Only four patients (1\%) contracted the disease through intravenous drug use. In 124 (30\%) patients, the mode of transmission was not determined. The mean CD4 lymphocyte count was 363 cells/ $\mathrm{mm}^{3}( \pm 95)$, the mean HIV-RNA count was 44,475 copies $/ \mathrm{mL}$ ( \pm 40,369), and 379 patients (92\%) were on HAART. Persistent proteinuria was encountered in 23 patients $(5.6 \%, 95 \% \mathrm{CI}=$ $3.6 \%$ to $8.3 \%$ ), and serum creatinine above $1.3 \mathrm{mg} / \mathrm{dL}$ was detected in 17 (4\%) patients (Table 1).

The variables black race, CD4 counts $<200$ cells $/ \mathrm{mm}^{3}$, HIV RNA $>100,000$ copies/mL and treatment with HAART were analyzed in subjects with and without proteinuria. In the blackrace group, 8 of 23 patients (35\%) had proteinuria and 146 of 388 patients $(37.6 \%)$ did not $(\mathrm{p}=0.784)$. Among the patients with CD4 levels $<200$ cells $/ \mathrm{mm}^{3}$, 9 of 22 patients (41\%) presented proteinuria and 69 of 308 patients (22.4\%) did not $(\mathrm{p}<0.048)$. Among the subjects with HIV RNA levels $>100,000$ copies/mL, 2 of 22 patients (9\%) presented proteinuria and 35 of 285 patients $(10.9 \%)$ did not $(\mathrm{p}=0.787)$, and in the group of patients on HAART, 22 of 379 (8\%) presented proteinuria and $92 \%$ did not $(p=1.0$, Table 2$)$.

The 23 patients with confirmed proteinuria were separated into three groups according to their Up/Uc levels: 16 (69.6\%) were discovered to have a $\mathrm{Up} / \mathrm{Uc}$ ratio < 1.0 ; five (22\%) patients had a $\mathrm{Up} / \mathrm{Uc}$ ratio between 1.0 and 3.0 and two (8.7\%) patients had a Up/Uc $>3.0$.

\section{Patients with a $\mathrm{Up} / \mathrm{Uc}<1.0$}

In the group of patients with $\mathrm{Up} / \mathrm{Uc}<1.0,16$ had ratios varying between 0.3 and 0.96 . Seven of these subjects (44\%) had CD4 levels of $<200$ cells $/ \mathrm{mm}^{3}$ and one $(6.3 \%)$ had a viral load of $>100,000$ copies $/ \mathrm{mL}$. Three patients had serum creatinine $>1.3 \mathrm{mg} / \mathrm{dL}$ (patients 1, 5 and 11, Table 3), and four had a history of systemic arterial hypertension (patient 5 , Table 3, had poorly-controlled hypertension). Only one patient was not receiving HAART and 10 received other medications (Table 3).

Patient 13 was a white male, age $57 \mathrm{y}$, who presented with nephrotic syndrome (10.9 g proteinuria), had a kidney biopsy, and the histological diagnosis was compatible with membranoproliferative glomerulonephritis (MPGN). This patient had negative tests for HBsAg, anti HCV, FAN, C3, C4, VDRL, ANCA and Cryoglobulin. He had a remission of proteinuria after beginning HAART and when last tested, had a Up/Uc ratio of 0.33 .

Patients with Up/Uc between 1.0 and 3.0

Five patients presented with a Up/Uc of between 1.0 and 3.0. One had a CD4 level of $<200$ cells $/ \mathrm{mm}^{3}$ and another had an HIV RNA of $>100,000$ copies/mL. One patient had a longstanding history of primary hypertension, and another was positive for anti HCV (Table 4). One patient in this group (patient 3, Table 4), a dark-skinned male, 51 years of age, developed nephrotic syndrome in March 2002. He had a 24hour proteinuria of $11.2 \mathrm{~g}$ and renal insufficiency, with maximum 
Table 2. Evaluation of patients with and without proteinuria according to the characteristics black race, CD4 levels of $<200$ cells/ $\mathrm{mm}^{3}$, HIV RNA $>100,000$ copies/mL and on HAART

\begin{tabular}{|c|c|c|c|c|c|c|c|}
\hline & With proteinuria & $\%$ & Without proteinuria & $\%$ & Total & $\%$ & pvalue \\
\hline Black race & 8 & 34.8 & 146 & 37.6 & 154 & 100 & 0.784 \\
\hline Not black race & 15 & 65.2 & 242 & 62.3 & 257 & 100 & \\
\hline CD4<200cells/mL & 9 & 40.9 & 69 & 22.4 & 78 & 100 & 0.048 \\
\hline CD4>200cells/mL & 13 & 59.1 & 239 & 77.6 & 254 & 100 & \\
\hline HIV RNA > 100,000counts $/ \mathrm{mm}^{3}$ & 2 & 9.1 & 35 & 10.9 & 37 & 100 & 0.787 \\
\hline HIV RNA $<100,000$ counts $/ \mathrm{mm}^{3}$ & 20 & 90.9 & 285 & 89.1 & 305 & 100 & \\
\hline Using HAART & 22 & 95.6 & 357 & 92 & 379 & 100 & 1.0 \\
\hline Not using HAART & 1 & 4.4 & 31 & 8 & 32 & 100 & \\
\hline
\end{tabular}

CD4=lymphocyte CD4 count (cells/mm³); HIV RNA count (copies/mL).

Table 3. Patients with urinary protein-creatinine ratio $(\mathrm{Up} / \mathrm{Uc})<1.0$

\begin{tabular}{|c|c|c|c|c|c|c|c|c|c|c|}
\hline Sex & Age & $\mathbf{R}$ & S Cr & Up/Uc & CD4 & HIVRNA & HAART & HBsAg & HCV & Other diseases \\
\hline M & 33 & DS & 1.8 & 0.34 & 186 & 400 & 3tc/d4t/efv/saq/rtv* & Neg & Neg & Hypertension \\
\hline M & 49 & DS & 0.9 & 0.46 & 542 & 400 & 3tc/azt/nfv* & Neg & Neg & - \\
\hline M & 40 & $\mathrm{~B}$ & 1.3 & 0.42 & 197 & 74700 & azt/ddi/nfv & - & - & - \\
\hline M & 42 & B & 1 & 0.49 & 70 & 400 & 3tc/azt/efv/nfv* & - & Neg & - \\
\hline M & 60 & B & 1.7 & 0.34 & 466 & 400 & 3tc/azt/nfv & - & - & Hypertension \\
\hline M & 25 & $\mathrm{~B}$ & 1.1 & 0.85 & 155 & 3100 & D4t/ddi/efz & - & - & - \\
\hline M & 33 & DS & 0.8 & 0.62 & 323 & 400 & $3 \mathrm{tc} / \mathrm{d} 4 \mathrm{t} / \mathrm{lpv}+\mathrm{rtv}^{*}$ & - & - & - \\
\hline M & 38 & DS & 1.1 & 0.33 & 118 & 44000 & 3tc/d4t/saq/rtv* & Neg & Neg & - \\
\hline M & 43 & DS & 0.9 & 0.42 & 367 & 65100 & 3tc/azt/ind/saq & Neg & Neg & Hypertension \\
\hline M & 46 & $\mathrm{~W}$ & - & 0.5 & 851 & 1420 & azt/3tc/Ind* & Neg & Neg & Hypertension \\
\hline M & 38 & DS & 1.5 & 0.45 & 63 & 400 & 3tc/d4t/lpv/rtv* & Neg & Neg & - \\
\hline M & 26 & W & 1.1 & 0.41 & - & - & azt/3tc/ind & - & - & - \\
\hline M & 57 & W & - & 0.3 & 431 & 400 & 3tc/d4t/efv & Neg & Neg & MPGN \\
\hline $\mathrm{F}$ & 37 & B & 0.6 & 0.7 & 275 & 17500 & None & Neg & Neg & - \\
\hline $\mathrm{F}$ & 37 & B & 0.5 & 0.96 & 255 & 400 & 3tc/d4t/efz & Neg & - & - \\
\hline M & 37 & DS & 0.9 & 0.64 & 26 & 208000 & 3tc/d4t/saq/rtv* & - & Neg & - \\
\hline
\end{tabular}

TMP/SMX=Trimethoprim-sulfamethoxasole. $\mathrm{M}=$ male; $\mathrm{F}=$ female; R=race; W=white; $\mathrm{DS}=$ dark skinned; $\mathrm{B}=\mathrm{black}$; $\mathrm{S}$ Cr=serum creatinine (mg/dL); CD4=lymphocyte CD4 count (cells/mm³); HIV RNA count (copies/mL); HAART=high active antiretroviral therapy; 3tc=lamivudina; d4t=stavudine; efv=efavirenz; saq=saquinavir; rtv=ritonavir; azt=zidovudine; nfv=nelfinavir; ddi=didanozine; ind=indinavir; lpv=lopinavir; MPGN=membranoproliferative glomerulonephritis; Neg=negative.

Table 4. Patients with urinary protein-creatinine ratio (Up/Uc) 1.0-3.0

\begin{tabular}{cccccccllll}
\hline Sex & Age & R & S Cr & Up/Uc & CD4 & HIVRNA & HAART & HBsAg & HCV & Other diseases \\
\hline M & 45 & DS & 1.2 & 2 & 98 & 18200 & 3tc/azt/efz* & - & - & Hypertension \\
M & 42 & DS & 1.1 & 1.3 & 202 & 299000 & 3tc/d4t/efv/ lpv/rtv & Neg & Neg & \\
M & 51 & DS & 1.5 & 2.1 & 678 & $<400$ & 3tc/azt/efz & Neg & Neg \\
M & 47 & B & 2.2 & 1.4 & 254 & $<400$ & 3tc/d4t/lvp+rtv & Neg & Neg & \\
F & 36 & W & 0.9 & 1.2 & 591 & $<400$ & azt/nvp/ind & Neg & Pos & \\
\hline
\end{tabular}

*TMP/SMX=Trimethoprim-sulfamethoxasole. $\mathrm{M}=$ =male; F=female; R=race; W=white; DS=dark skinned; B=black; S Cr=serum creatinine (mg/dL); CD4=lymphocyte CD4 count (cells/ $\mathrm{mm}^{3}$ ); HIV RNA count (copies/mL); HAART=highly active antiretroviral therapy; 3tc=lamivudine; $\mathrm{d} 4 \mathrm{t}=$ =stavudine; efv=efavirenz; $\mathrm{rtv}=$ ritonavir; azt=zidovudine; $\mathrm{nvp}=$ nevirapine; ind=indinavir; lpv=lopinavir.

Table 5. Patients with urinary protein-creatinine ratio $(\mathrm{Up} / \mathrm{Uc})>3.0$

\begin{tabular}{ccccccccccl}
\hline Sex & Age & R & S Cr & Up/Uc & CD4 & HIVRNA & HAART & HBsAg & HCV & Other diseases \\
\hline M & 24 & B & 2.3 & 11 & 171 & $<400$ & 3tc/d4t/efv* & Neg & Neg & FSGS \\
M & 38 & DS & 1.2 & 3.8 & 468 & $<400$ & 3tc/d4t/efz & Pos & Neg & Inconclusive \\
\hline
\end{tabular}

*TMP/SMX=Trimethoprim-sulfamethoxasole. $\mathrm{M}=$ male; $\mathrm{F}=$ female; $\mathrm{R}=$ race; $\mathrm{DS}=$ dark skinned; $\mathrm{B}=$ =black; S Cr=serum creatinine (mg/dL); CD4=lymphocyte CD4 count (cells/mm³); HIV RNA count (copies/mL); HAART=highly-active antiretroviral therapy; 3tc=lamivudine; d4t=stavudine; efv=efavirenz; FSGS=focal segmental glomerulosclerosis; Neg=negative; Pos=positive. 
serum creatinine of $3.7 \mathrm{mg} / \mathrm{dL}$. He had negative tests for HBsAg, anti HCV, FAN, C3, C4, VDRL, ANCA and Cryoglobulin. HAART therapy and prednisone (1 mg/Kg/day) was begun, and after 10 months his Up/Uc had decreased to 2.1 and serum creatinine to $1.5 \mathrm{mg} / \mathrm{dL}$.

\section{Patients with Up/Uc > 3.0}

Only two patients had Up/Uc ratios above 3.0, and they had a kidney biopsy (Table 5). The first patient, a male, 24 years of age and black, presented with a CD4 level of 171 cells/mm³ HIV RNA was undetectable, and HBsAg and HCV tests were negative. Kidney histology was compatible with classic HIVAN, or the patient had FSGS, with cystic dilatation of tubules and slight interstitial fibrosis under the microscope. Immunofluorescence indicated an absence of glomerular deposits and the presence of tubular casts with IgA and IgM. There was no evidence of glomeruli under electron-microscope analysis.

A second male patient, 38 years old and dark-skinned, presented CD4 levels of 468 cells $/ \mathrm{mm}^{3}$, an undetected viral load, a positive result for HBsAg and negative for HCV. Renal biopsy revealed three normal glomeruli, with tubular degeneration and slight interstitial fibrosis, an absence of glomerular deposits with immunofluorescence and degenerative podocyte alterations on electron-microscope analysis. Tubuloreticular inclusions were not observed.

\section{Discussion}

In our study, the prevalence of persistent proteinuria was $5.6 \%$, which is low when compared with other studies. In 1993, Kimmel et al. studied 505 patients in Washington (USA) and encountered proteinuria in 29.8\%; in 2001, Hailemariam et al. in Zurich (Switzerland), reported a prevalence of 18\% (among 239 patients), and Crowley et al., in 2001 in New Haven (USA), described persistent proteinuria in $14 \%$ of 49 patients [19-21]. However, Ahuja et al. found proteinuria in $6.8 \%$ of 557 patients studied in Texas (USA) in 1999 [22]. This variation appears to reflect distinct situations. The former studies involved referred-nephrology patients; one of the studies was done prior to HAART use, which was introduced into clinical practice in 1996. The later study was similar to our investigation, since it involved an entire population with HIV/AIDS, nearly all of who had been receiving HAART.

Other factors may also be responsible for the lower prevalence of persistent proteinuria, the first of which is black race. In our study group, only 37.5\% (154 patients) were black, a larger proportion (44.3\%) were considered dark-skinned.

HIVAN has been described almost exclusively within the black race group. This finding suggests that there may be genetic factors that are associated with race. Freedman et al., in 1999, compared 201 black patients who developed endstage renal-disease related (ESRD) to HIVAN to 50 positive black patients without renal disease. He found a family history of renal disease in $24.4 \%$ of the patients who developed ESRD, compared to $6 \%$ in the control group [23]. We found no significant differences related to black race and proteinuria. Possibly, the mixed race factor, in this case involving the darkskinned patients, is a protective factor against HIVAN. The only patient within our sample who presented classic symptoms of HIVAN was black.

Another factor relevant for explaining the low prevalence of proteinuria in our study could be the use of HAART. Almost all patients (92.2\%) were on HAART, which was evident from the good control of the viral load, since only $11 \%$ (37 patients) of this group had a viral loads $>100,000$ copies $/ \mathrm{mL}$. Nevertheless we found no significant differences in proteinuria between patients using HAART versus those that did not. However, very few patients were not using HAART, which makes it difficult to make an objective comparison. However, we found evidence that HAART reduces renal damage caused by HIV-1; two patients controlled their renal diseases by using HAART. One of them had a $24 \mathrm{~h}$ proteinuria of $11.2 \mathrm{~g}$ and creatinine levels of $3.7 \mathrm{mg} / \mathrm{mL}$, in 2002, which decreased to a $\mathrm{Up} / \mathrm{Uc}$ of 2.1 and creatinine levels of $1.5 \mathrm{mg} / \mathrm{dL}$ in 2004. This provides strong evidence that HAART is a protective factor in the development of HIVAN; possibly, it could stabilize the progression of renal disease. Cosgrove et al. found that patients on HAART presented higher lymphocyte CD4 counts, lower viral load levels and considerably lower levels of proteinuria, in comparison to those not on medication [24].

A CD4 count below 200 cells $/ \mathrm{mm}^{3}$ was the only risk factor significantly associated with proteinuria $(\mathrm{p}<0.048)$. However, conflicting results are described in literature. While association of a CD4 level of $<200$ cells $/ \mathrm{mm}^{3}$ and HIVAN is a consensus, its association with proteinuria is not so clear. In 2001, Crowley et al. evaluated 67 HIV-positive patients; they were unable to find an association of proteinuria with CD4 levels below 200 cells $/ \mathrm{mm}^{3}$. Moreover, they did not encounter differences in the viral-load levels when comparing groups with and without proteinuria [21]. On the other hand, Szczech et al. in 2002, in a multicentric study of 2057 HIV-positive women, encountered a higher frequency of proteinuria in patients with CD4 levels below 200 cells $/ \mathrm{mm}^{3}$ [25].

A high proportion of the patients who presented proteinuria and CD4 levels $<200$ cells/mL, were in the group with $\mathrm{Up} / \mathrm{Uc}<$ 1.0. They were also taking other medications besides HAART, especially trimethoprim/sulfamethoxazole. Frequently, levels of proteinuria lower than $1.5 \mathrm{~g}$ per day are of tubular origin and could possibly be related to drugs that are a potential cause of interstitial nephritis [26]. So, one could state that proteinuria in this group was not necessarily linked to HIVAN; it was also related to other causes, including other infections and drugs, resulting in great variability in its frequency. The other patient with Up/Uc $>3.0$ (patient 2, Table 5) also presented co-infection with hepatitis B virus, and a renal biopsy revealed podocytes with degenerative alterations. The analyzed fragment contained only three glomeruli, thus rendering it impossible to discard focused lesions, such as FSGS. This patient was not tested for the marker HBeAg (the " $e$ " antigen of hepatitis B), frequently found in patients with 
glomerulonephritis associated with hepatitis B. However, the main histological factors encountered in hepatitis-B-associated glomerulonephritis are membranous and membranoproliferative glomerulopathy, which the biopsy was able to discard $[27,28]$. Similarly to proteinuria, the prevalence of HIVAN seems to be linked to the characteristics of the group studied, especially considering the predominance of HAART. Studies during the 80s and at the beginning of the 90s illustrated a prevalence of HIVAN in the black population of around $10 \%$, reaching $20 \%$ in certain reports $[2,3,29,30]$. Since 1996, with the introduction of HAART, this prevalence has gradually been reduced. In 1999, Ahuja et al. described a 3.5\% prevalence of HIVAN amongst the black race groups, the majority of who had been receiving HAART [22].

Two patients had changing patterns of renal disease in the HIV-1 infection. The first was included in the group with $\mathrm{Up} / \mathrm{Uc}<1.0$, (patient 13, Table 3). Two years before, in 2002, he presented a $24 \mathrm{~h}$ proteinuria of $10.9 \mathrm{~g}$, and the histological pattern showed membranoproliferative glomerulonephritis. After going on HAART, he came into remission. By the end of the study he had a Up/Uc of 0.3. The second patient had his renal disease diagnosed two years previously, (patient 3, Table 4). His disease went through partial remission, during which renal function improved considerably after beginning HAART and a course of cortisone. Unfortunately, there was no histological confirmation of the renal lesion in this case. The two patients who presented proteinuria remission underwent other tests for secondary causes of glomerulopathy, which proved negative.

Several studies have also report a reduction in the levels of proteinuria and an improvement in renal function after HAART. Some characteristics are common in these reports, especially the predominance of black race, high levels of immune suppression or advanced stages of acquired immunedeficiency syndrome, illustrated by CD4 lymphocyte levels of $<200$ cells $/ \mathrm{mm}^{3}$ and high viral-load levels (over 750,000 copies/mL), and the introduction of HAART during the initial stages of the renal lesion [31-34].

The reason for the improvement of histological lesions in certain patients and not in others is not completely clear. It may be related to the moment that the patient started receiving HAART. The renal lesions progress in a sequential manner, beginning with interstitial inflammation, microcystic dilation, and finally irreversible interstitial fibrosis. The introduction of treatment during very early stages of disease evolution may help to improve the prognosis of glomerular alterations [35].

Although the prevalence of HIVAN was low in our sample, once renal disease has becomes established, it provokes a quick and progressive loss of renal function. Early diagnosis is not a simple matter, since the glomerular lesion associated with HIV rarely presents clinical signs. The intensity of the tubular-interstitial lesions is responsible for the nephropathy salt loss, which is why these patients do not present serious edemas, in spite of the high proteinuria. We conclude that testing for persistent proteinuria and determining an early diagnosis of HIVAN should become routine practice for HIV/ AIDS patients. Finally, we suggest that HAART could revert some renal lesions caused by HIV.

\section{References}

1. AIDS epidemic update: special report on HIV/AIDS: December 2006 - (www.unaids.org).

2. Rao T.K.S., Filippone E.J., Nicastri A.D., et al. Associated focal and segmental glomerulosclerosis in AIDS. N Engl J Med 1984;310:669-73.

3. Pardo V., Aldana M., Colton R.M., et al. Glomerular lesions: The acquired immunodeficiency syndrome. Ann Intern Med 1984;101:429-34.

4. Rao T.K.S. Clinical features of human immunodeficiency virus associated nephropathy. Kidney Int 1991;40:S13-8.

5. Kimmel P.L., Phillips T.M., Ferreira-Centeno A., et al. Idiotypic IgA Nephropathy In Patients With Human Immunodeficiency Virus Infection. N Eng J Med 1991;327:702-6.

6. Casanova S., Mazzucco G., Barbiano di Belgiojoso G., et al. Pattern of glomerular involvement in human immunodeficiency virusinfected patients: A Italian study. Am J Kidney Dis 1995;26:446-53.

7. D'Agati V., Appel G.B. HIV infection and the kidney. J Am Soc Nephrol 1997;138-52.

8. Rivera M., Gonzalo A., Mampaso F., et al. The heterogeneity of glomerulonephritis associated with HIV. Nephrol Dial Transplant 1999;14:244-5.

9. Haas M., Rajaraman S., Ahuja T., et al. Fibrillary/Immunotactoid glomerulonephritis in HIV-positive patients: a report of three cases. Nephrol Dial Transplant 2000;15:1679-83.

10. Martin J.L., Thomas D., Colindres R.E. Imunotactoid Glomerulopathy in na HIV-Positive African-American Man. Am J Kidney Dis 2003;42:6-10.

11. D’Agati V., Suh J.L., Carbone L., et al. Pathology of HIV-associated nephropathy: a detailed morphologic and comparative study. Kidney Int 1989;35:1358-70.

12. Cohen A.H. HIV-associated nephropathy: current concepts. Nephrol Dial Transplant 1998;13:540-2.

13. Olantinwo T., Hewitt R.G., Venuto R.C. Human Immunodeficiency Virus-Associated Nephropathy. Arch Intern Med 2004;164:333-6.

14. Winston J.Á., Klotman M.E., Klotman P.E. HIV-associated nephropathy is a late, not early, manifestation of HIV-1 infection. Kidney Int 1999;55:1036-40.

15. Szczech L.A., Gupta S.K., Habash R., et al. The clinical epidemiology and course of the spectrum of renal diseases associated with HIV infection. Kidney Int 2004;66:1145-52.

16. Gardner L.I., Holmberg S.D., Williamson J.M., et al. Development of proteinuria or elevated serum creatinine and mortality HIV-infected women. J Acquir Immune Defic Syndr 2003;32(2):203-9.

17. Gupta S.K., Mamlin B.W., Johnson C.S., et al. Prevalence of proteinuria and the development of chronic kidney disease in HIV-infected patients. Clin Nephrol 2004;61(1):1-6.

18. Lopes G.S., Marques L.P.J., Rioja L.S., et al. Glomerular Disease and Human Immunodeficiency Virus Infection in Brazil. Am J Nephrol 1992;12:281-7.

19. Kimmel P.L., Umana W.O., Bosch J.P. Abnormal urinary protein in HIV-infected patients. Clin Nephrol 1993;39:17-21.

20. Hailemariam S., Walder M., Burger H.R., et al. Renal pathology and premortem clinical presentation of Caucasian patients with AIDS: An autopsy study from the era prior to antiretroviral therapy. Swiss Med Wkly 2001;131:412-7.

21. Crowley S.T., Cantwell B., Abu-Alfa A., et al. Prevalence of persistent asymptomatic proteinuria in HIV-infected outpatients and lack of correlation with viral load. Clin Nephrol 2001; $55: 1-6$. 
22. Ahuja T.S., Boruckia M., Funtanilla M., et al. Is the Prevalence of HIV-Associated Nephropathy Decreasing? Am J Nephrol 1999;19:655-9.

23. Freedman B.I., Soucie M., Stone S.M., et al. Familial Clustering of End-Stage Renal Disease in Blacks With HIV-Associated Nephropathy. Am J Kidney Dis 1999;34:254-8.

24. Cosgrove C.J., Abu-Alfa A.K., Perazella M.A. Observations on HIV-Associated Renal Disease in the Era of Highly Active Antiretroviral Therapy. Am J Med Sci 2002;323(2):102-6.

25. Szczech L.A., Gange S.J., Van der Horst, et al. Predictors of proteinuria and renal failure among women with HIV infection. Kidney Int 2002;61:195-202.

26. Rao T.K.S. Acute Renal Failure Syndromes in Human Immunodeficiency Virus Infection. Semin Nephrol 1998; $18: 378-95$.

27. Jonhson R.L., Couser W.G. Hepatitis B infection and renal disease: Clinical, immunopathogenetic and therapeutic consideration. Kidney Int 1990;37:663.

28. Lai K.N., Lai F.M. Clinical features and the natural course of hepatitis B virus-related glomerulopathy in adults. Kidney Int 1991;35:S40-5.
29. Gardenswartz M.H., Lerner C.W., Seligson G.R., et al. Renal disease in patients with AIDS: A clinicopathologic study. Clin Nephrol 1984;21:197-204.

30. Langs C., Gallo G.R., Schacht R.G., et al. Rapid renal failure in AIDS-associated focal glomerulosclerosis. Arch Intern Med 1990; $150: 287-92$.

31. Wali R.K., Drachenberg C.I., Papadimitriou J.C., et al. HIVassociated nephropathy and response to highly-active antiretroviral therapy. Lancet 1998;352:783-4.

32. Chemlal K., Nocky D., Kenouch S., et al. Dramatic Improvement of Renal Dysfunction in a Human Immunodeficiency Virus Infected Woman Treated with Highly Active Antiretroviral Therapy. Clin Infect Dis 2000;31:805-6.

33. Kirchner J.T. Resolution of renal failure after initiation of HAART: 3 cases and a discussion of the literature. AIDS Reader 2002;12:103-5.

34. Scheurer D. Rapid reversal of renal failure after initiation of HAART: a case report. AIDS Reader 2004;14:443-7.

35. Rodriguez R.A. Effect of HAART on HIVAN - What can the clinician expect? AIDS Read 2004;14:443-8. 